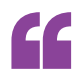

the findings could have important implications for the development of ... new therapeutic interventions

$\Rightarrow$ NEURODEGENERATIVE DISEASE

\title{
Proteome points to synaptic dysfunction in dementia
}

Changes in the levels of specific synaptic proteins could provide a unique fingerprint to enable early diagnosis of several types of dementia, according to a new proteomic study carried out in human brain samples. The results also show that certain synaptic proteins might present a target for future therapies for dementia.

Neurodegenerative diseases such as Alzheimer disease (AD) and dementia with Lewy bodies (DLB) are characterized by cognitive decline, which is associated with a loss of synapses. This synaptic loss is thought to precede neuronal degeneration, and correlates more closely with cognitive dysfunction than do pathological hallmarks such as amyloid- $\beta(A \beta)$ and tau, indicating that synaptic dysfunction represents an important therapeutic target.

To better understand the nature of the synaptic changes that take place in dementia, Erika Bereczki and colleagues carried out a systematic, quantitative proteome comparison between 32 post-mortem brain samples taken from individuals who had AD, Parkinson disease dementia (PDD) or DLB, or were neurologically healthy.

"The complex structural and functional organization of the brain warrants the application of systematic approaches," explains Bereczki. "We have taken advantage of technological advances in proteomics analyses that provide high-throughput screening."

The team identified 10,325 proteins that were differentially regulated across the patient groups, 851 of which were synaptic proteins. Twenty-five synaptic proteins exhibited significantly different concentrations in patients with dementia and neurologically normal controls.

A multivariate analysis showed that the different levels of synaptic proteins enabled discrimination not only between individuals with and without dementia, but also between individuals with $\mathrm{AD}$ and individuals with PDD. Intriguingly, a reduction in the levels of certain synaptic proteins correlated with a rapid decline in Mini-Mental State Examination (MMSE) score and a low final MMSE score before death, as well as with the degree of $A \beta$ or $\alpha$-synuclein pathology in patient brains.

The investigators suggest that the results support the presence of shared synaptic pathogenic mechanisms among these different forms of dementia. In addition, the team think that the findings could have important implications for the development of prognostic and diagnostic biomarkers, as well as new therapeutic interventions. "We believe that pinpointing overall alterations of synaptic proteins occurring in dementia patients brings us one step closer to identifying a disease-specific biological target for dementia prevention and therapy," says Bereczki.

Charlotte Ridler

ORIGINAL ARTICLE Bereczki, E. et al. Synaptic markers of cognitive decline in neurodegenerative diseases: a proteomic approach. Brain https://doi. org/10.1093/brain/awx352 (2018) 